\title{
Magneto-Coulomb Drag: Interplay of Electron-Electron Interactions and Landau Quantization
}

Bønsager, Martin Christian; Flensberg, Karsten; Hu, Ben Yu-Kuang; Jauho, Antti-Pekka

Published in:

Physical Review Letters

Link to article, DOI:

10.1103/PhysRevLett.77.1366

Publication date:

1996

Document Version

Publisher's PDF, also known as Version of record

Link back to DTU Orbit

Citation (APA):

Bønsager, M. C., Flensberg, K., Hu, B. Y-K., \& Jauho, A-P. (1996). Magneto-Coulomb Drag: Interplay of Electron-Electron Interactions and Landau Quantization. Physical Review Letters, 77(7), 1366-1369. https://doi.org/10.1103/PhysRevLett.77.1366

\section{General rights}

Copyright and moral rights for the publications made accessible in the public portal are retained by the authors and/or other copyright owners and it is a condition of accessing publications that users recognise and abide by the legal requirements associated with these rights.

- Users may download and print one copy of any publication from the public portal for the purpose of private study or research.

- You may not further distribute the material or use it for any profit-making activity or commercial gain

- You may freely distribute the URL identifying the publication in the public portal 


\title{
Magneto-Coulomb Drag: Interplay of Electron-Electron Interactions and Landau Quantization
}

\author{
Martin Christian Bønsager, ${ }^{1,2}$ Karsten Flensberg, ${ }^{1,3}$ Ben Yu-Kuang Hu, ${ }^{1}$ and Antti-Pekka Jauho ${ }^{1}$ \\ ${ }^{1}$ Mikroelektronik Centret, Bygn. 345, Danmarks Tekniske Universitet, DK-2800 Lyngby, Denmark \\ ${ }^{2}$ Department of Physics, Indiana University, Bloomington, Indiana 47405-4202 \\ ${ }^{3}$ Dansk Institut for Fundamental Metrologi, Bygn. 307, Anker Engelunds Vej 1, DK-2800 Lyngby, Denmark
}

(Received 4 March 1996)

\begin{abstract}
We use the Kubo formalism to calculate the transresistivity $\rho_{21}$ for carriers in coupled quantum wells in a large perpendicular magnetic field $B$. We find that $\rho_{21}$ is enhanced by approximately 50-100 times over that of the $B=0$ case in the interplateau regions of the integer quantum Hall effect. The presence of both electron-electron interactions and Landau quantization results in (i) a twin-peaked structure of $\rho_{21}(B)$ in the interplateau regions at low temperatures and (ii) for the chemical potential at the center of a Landau level band, a peaked temperature dependence of $\rho_{21}(T) / T^{2}$. [S0031-9007(96)00875-7]
\end{abstract}

PACS numbers: 73.50.Dn, 73.20.Mf

The combination of electron-electron $(e-e)$ Coulomb interaction and strong magnetic $(B)$ field in two-dimensional electron gases (2DEGs) has provided an exciting venue of research for both experimentalists and theorists over the past few decades [1]. One well-known example of this is the fractional quantum Hall effect, where the physics is determined by the subtle interplay between the interactions and the large density of states (DOS) caused by all the electrons being confined to the lowest Landau level (LL). Even in the integer quantum Hall effect, where $e-e$ interactions do not play such a crucial role, they are thought to determine some important factors such as the position of the edge currents [2]. On the other hand, $e-e$ interactions in parabolic confined systems in a magnetic field surprisingly have no effect on cyclotron resonance measurements (due to the generalized Kohn's theorem) [3]. Thus phenomena involving interelectron interactions in a $B$ field often produce surprising and interesting results.

Recently there have been many experiments on coupled 2DEGs electron systems which have probed the effect of Coulomb interactions, both with and without magnetic field. Some experiments measured tunneling from one well to the other [4], while in others the quantum wells were separated by a distance at which interwell tunneling was negligible but interwell Coulomb interactions were experimentally detectable [5]. The latter "drag" experiments, so called because one drives a current in one layer and measures the consequence of the frictional drag due to the interlayer interactions in the second layer, provide a direct measure of the interwell Coulomb interaction. Not surprisingly, physics centered around drag phenomena has generated many theoretical investigations [6-12]. In principle, drag experiments should provide a unique forum for exploring the subtleties of the interplay of $e-e$ interactions in a magnetic field. Thus far, however, only zero magnetic field data have been published.

In this Letter, we present a first-principles formulation of the drag problem in a magnetic field, including effects due to weak impurity scattering, starting from Kubo theory. We then show results of an explicit numerical calculation of the transresistivity $\rho_{21}^{x x}$ for short-ranged scatterers in the interplateau regions of the integer quantum Hall regime. We demonstrate that the aforementioned large DOS and screening due to intralayer $e-e$ interactions have profound effects on $\rho_{21}$.

In principle, a drag experiment can be performed by imposing a fixed electric field $\mathbf{E}_{1}$ on the "drive" layer (henceforth called layer 1) and measuring the current $\mathbf{J}_{2}$ dragged along in the "response" layer (called layer 2), placed a distance $d$ away. The Kubo formalism allows one to compute the transconductivity $\overleftrightarrow{\sigma}_{i j} \mathbf{E}_{j}=\mathbf{J}_{i}$ $(i=1,2)$, which we can invert to obtain the transresistivity $\stackrel{\leftrightarrow}{\rho}_{i j} \mathbf{J}_{j}=\mathbf{E}_{i}$ [13]. For time-independent transport, to second (i.e., lowest nonvanishing) order in the screened interlayer interaction $W_{12}(q, \omega), \stackrel{\leftrightarrow}{\sigma}_{21}$ is given by $[10,11,14]$

$$
\begin{aligned}
\sigma_{21}^{\alpha \beta}= & \frac{e^{2}}{2 \hbar^{3}} \int \frac{d \mathbf{q}}{(2 \pi)^{2}} \int_{-\infty}^{\infty} \frac{d \omega}{2 \pi}\left|W_{12}(q, \omega)\right|^{2} \\
& \times\left[-\frac{\partial n_{B}(\hbar \omega)}{\partial \omega}\right] \Delta_{2}^{\alpha}\left(\mathbf{q}, \mathbf{q} ; \omega+i 0^{+}, \omega-i 0^{+}\right) \\
& \times \Delta_{1}^{\beta}\left(-\mathbf{q},-\mathbf{q} ;-\omega-i 0^{+},-\omega+i 0^{+}\right),
\end{aligned}
$$

where $n_{B}$ is the Bose function and $\boldsymbol{\Delta}_{l}$ is the imaginarytime Fourier transform of the thermal-averaged correlation function $i\left\langle\mathcal{T}_{\tau} \mathbf{j}_{l}(\mathbf{q}=0, \tau=0) \rho_{l}(\mathbf{q}, \tau) \rho_{l}\left(-\mathbf{q}, \tau^{\prime}\right)\right\rangle$ $[10,11]$. Screening is calculated using the random phase approximation for electrons in a magnetic field with weak impurity scattering [15], where the density-response function $\chi(q, \omega)$ is given diagrammatically by Fig. 1(a). We assume throughout this paper that there are like charges in both layers (generalization to unlike charges is straightforward), and that spin splitting is negligible [16].

We let $x-y$ be the confinement plane for the electrons $\mathbf{B}=B \hat{z}$, and use the Landau gauge $\mathbf{A}=(0, B x, 0)$. $\Delta\left(\mathbf{q}, \mathbf{q} ; \omega \pm i 0^{+}, \omega \mp i 0^{+}\right)$is a real, gauge invariant quantity. Ignoring diagrams with crossed impurity lines (which are negligible in the weak scattering and high $B$ field limit), $\Delta$ is shown diagrammatically in Fig. 1(b) and can be written down in terms of the single-particle Green 


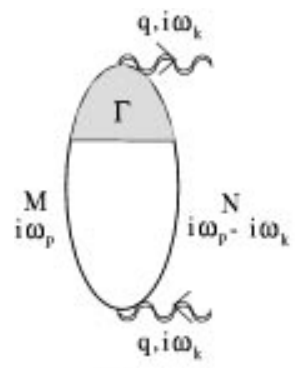

(a)

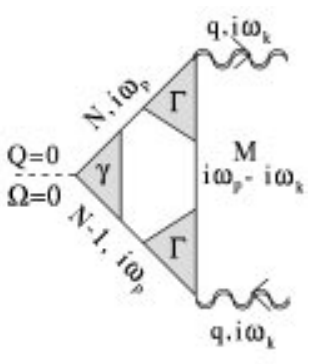

(b)
FIG. 1. (a) The diagram corresponding to the density response function $\chi$. (b) The triangle diagram contribution to the threebody response function $\Delta$. The $\Gamma$ and $\gamma$ are the charge and current vertices, respectively, and the labels $M$ and $N$ denote the LL's of the impurity-broadened Green functions.

functions $G$, and charge and current vertices. For 2DEGs electrons in a quantizing magnetic field it is crucial to include the impurity effects from the outset to avoid nonphysical results. We do this within the self-consistent Born approximation (SCBA) [15]. Then the Green functions and the self-energy $\Sigma$ depend only on the LL in$\operatorname{dex}[15], G\left(N, i \omega_{n}\right)=\left[i \omega_{n}-\varepsilon_{N}-\Sigma\left(N, i \omega_{n}\right)\right]^{-1}$. In calculating $\Delta$, we include all ladder-type diagrams as required by the Ward identity.

The general expression for $\boldsymbol{\Delta}$ for arbitrary scattering is complicated, but many simplifications occur in the weak-scattering limit $\omega_{c} \tau \gg 1$, where $\omega_{c}=e B / m$ is the cyclotron frequency and $\tau$ is the Born approximation scattering time. In particular, it is possible to link $\Delta$ with the $\chi(\mathbf{q}, \omega)$ shown in Fig. 1(a) [17]. We find

$$
\begin{aligned}
& \Delta\left(\mathbf{q}, \mathbf{q} ; \omega \pm i 0^{+}, \omega \mp i 0^{+}\right)= \\
& \quad \pm 2 \hbar^{2} e^{-1} \mathbf{q} \times \mathbf{B} \frac{\operatorname{Im}[\chi(\mathbf{q}, \omega)]}{B^{2}}+O\left(\left(\omega_{c} \tau\right)^{-1}\right) .
\end{aligned}
$$

It is worth emphasizing that for $\omega_{c} \tau \gg 1$, the relationship between $\boldsymbol{\Delta}$ and $\operatorname{Im}[\chi]$ holds for arbitrary impurity scattering potential $U(\mathbf{q})$, whereas $\Delta(B=0)$ is related to $\operatorname{Im}[\chi]$ only for $q$-independent $U[11,12,18]$.

A brief discussion of the \pm sign occurring in the high field limit of Eq. (2) is appropriate. The Onsager relation and the vector nature of $\boldsymbol{\Delta}$ imply that it must have the form [19] $\boldsymbol{\Delta}\left(\mathbf{q}, \mathbf{q} ; \omega \pm i 0^{+}, \omega \mp i 0^{+}, \mathbf{B}\right)=$ $\mathbf{q} u(q, B, \omega) \pm(\mathbf{q} \times \mathbf{B}) v(q, B, \omega)$. The $\mathbf{q} u$ term dominates for small $B$, while the $(\mathbf{q} \times \mathbf{B}) v$ term dominates for $\omega_{c} \tau \gg 1$, which is consistent with Eq. (2). The form of $\boldsymbol{\Delta}$ implies from Eq. (1) that as $B$ is increased from $0, \sigma_{21}^{x x}$ changes sign at some point. Does this mean a change in sign of an experimentally measured quantity? If the measured quantity is the transresistivity $\rho_{21}^{x x}$, as is usually the case [5], the answer is no, for the following reason. In terms of $\stackrel{\leftrightarrow}{\sigma}_{i j}, \quad \stackrel{\leftrightarrow}{\rho}_{21}=\left[-\overleftrightarrow{\sigma}_{11} \stackrel{\leftrightarrow}{\sigma}_{21}^{-1} \stackrel{\leftrightarrow}{\sigma}_{22}+\stackrel{\leftrightarrow}{\sigma}_{12}\right]^{-1} \approx-\overleftrightarrow{\sigma}_{22}^{-1} \overleftrightarrow{\sigma}_{21} \stackrel{\leftrightarrow}{\sigma}_{11}^{-1}$ (since $\left.\left|\overleftrightarrow{\sigma}_{i i}\right| \gg\left|\overleftrightarrow{\sigma}_{21}\right|\right)$. For $B=0, \overleftrightarrow{\sigma}_{i i}$ are diagonal and $\rho_{21}^{x x}(B=0)=-\sigma_{21}^{x x} /\left(\sigma_{11}^{x x} \sigma_{22}^{x x}\right)$; i.e., $\rho_{21}^{x x}$ and $\sigma_{21}^{x x}$ have opposite signs. In contrast, for quantum Hall systems, $\left|\sigma_{i i}^{x y}\right| \gg \sigma_{i i}^{x x}$ and hence $\rho_{21}^{x x}\left(\omega_{c} \tau \gg 1\right) \approx-\sigma_{21}^{y y} / \sigma_{11}^{x y} \sigma_{22}^{y x}$. Since $\sigma_{11}^{x y} \sigma_{22}^{y x}<0$, this implies that $\sigma_{21}^{x x}\left(\omega_{c} \tau \gg 1\right)$ and $\rho_{21}^{x x}\left(\omega_{c} \tau \gg 1\right)$ have the same sign. Therefore, even though $\sigma_{21}^{x x}$ changes sign as $B$ increases, the experimentally relevant quantity $\rho_{21}^{x x}$ is negative in both cases. A physical explanation of this result is illustrated in Fig. 2.

Equations (1) and (2) form the basis of our numerical calculations. We obtain $\chi(q, \omega)$ by solving the appropriate vertex equation, and perform the integrals in (1) to obtain $\stackrel{\leftrightarrow}{\sigma}_{21}$ and consequently $\stackrel{\leftrightarrow}{\rho}_{21}$. We discuss the technical details elsewhere [19] and focus here on the qualitative features and some of the numerical results.

Without impurities, all electrons in a particular LL are degenerate. When the scattering is weak (so that inter-LL coupling can be ignored) within the SCBA with short-ranged scatterers [i.e., scattering interaction range $\ll \ell_{B} / \sqrt{2 N+1}$, where $\left.\ell_{B}=(\hbar / e B)^{1 / 2}\right] \Sigma\left(N, i \omega_{n}\right)$ is $N$ independent, and the electrons in a LL are distributed in bands where the DOS is semielliptical [15]. The DOS at the center of the LL is approximately $\sqrt{2 \omega_{c} \tau \pi^{-1}} g_{0}$, where $g_{0}=m \pi^{-1} \hbar^{-2}$ is the two-dimensional zero magnetic field DOS. Thus when $\omega_{c} \tau \gg 1$ (achieved in clean GaAs samples at fields under a tesla) the DOS is greatly enhanced over the $B=0$ value. The low-temperature transresistivity for fixed $T$ is to a first approximation directly proportional to the product of the thermally averaged DOS of both layers, $g_{1}(B) g_{2}(B)$, around the chemical potential $\mu$ (since the more phase space there is available for scattering around the Fermi surface, the larger the probability for interlayer momentum transfer). Hence one might expect that (1) $\left|\rho_{21}^{x x}\left(\omega_{c} \tau \gg 1\right)\right| \gg\left|\rho_{21}^{x x}(B=0)\right|$, and $(2) \rho_{21}^{x x}(B)$ would more or less simply reflect the shape of $g_{1}(B) g_{2}(B)$.

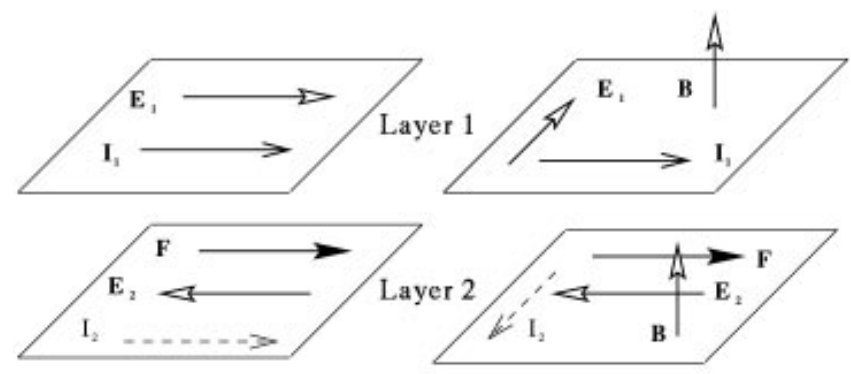

(a)

(b)

FIG. 2. Schematic illustration of the sign of the diagonal elements of the $\stackrel{\leftrightarrow}{\sigma}_{21}$ and $\stackrel{\leftrightarrow}{\rho}_{21}$ at (a) $B=0$ and (b) strong $B$ field (charges assumed positive). $\quad \mathbf{I}_{i}$ are the currents, $\mathbf{E}_{i}$ are electric fields, and $\mathbf{F}$ is the average net force transmitted from layer 1 to 2. For a transresistivity (transconductivity) measurement, $\mathbf{I}_{2}=0\left(\mathbf{E}_{2}=0\right)$ and $\mathbf{E}_{2}\left(\mathbf{I}_{2}\right)$ is measured. While $\sigma_{21}^{\alpha \alpha}$ changes sign going from (a) to (b), $\rho_{21}^{\alpha \alpha}$ does not. For transresistivity measurements, $\mathbf{E}_{2} \| \mathbf{F}$ because $\mathbf{F}-e\left(\mathbf{E}_{2}+\left\langle\mathbf{v}_{2}\right\rangle \times \mathbf{B}\right)=0$, and $\left\langle\mathbf{v}_{2}\right\rangle=0$. Hence, under conditions where $\mathbf{I}_{1} \| \mathbf{F}$, which is the case when there is inversion symmetry (e.g., when $B=0$ ), or when the electron distribution is a drifted Fermi-Dirac (e.g., when $\omega_{c} \tau \gg 1$ ), there is no Hall transresistivity. 
Figure 3 shows the results of a calculation for $\rho_{21}^{x x}(B)$ for two identical layers at fixed densities. For comparison, we also show $|\operatorname{Re}[\chi(q \rightarrow 0, \omega=0)]|=\partial n / \partial \mu \equiv$ $g$ [20]. As expected, $\rho_{21}^{x x}$ is very large; approximately 50-100 times larger than at $B=0$ [21]. Also $\rho_{21}$ is largest when $\mu$ is in the bands of extended states, and suppressed when it is in between the extended bands $[9,22]$. However, the shape of $\rho_{21}(B)$ is markedly different from $g^{2}(B)$. Relative to $g^{2}(B)$, there is an enhancement in $\rho_{21}(B)$ at the edges of the broadened LL and suppression at the center.

This effect originates from the screening properties of the system. Recall that $\rho_{21}$ also depends on interlayer coupling, which is given by the screened interlayer interaction $W_{12}$. Roughly, $\rho_{21}$ is proportional to $g_{1} g_{2}\left|W_{12}\right|^{2}$. For 2DEGs, the range of the screened interaction varies inversely with $g$ [15]. Therefore, increasing $g(B)$ weakens the interlayer coupling, implying that the terms $g_{1} g_{2}$ and $\left|W_{12}\right|^{2}$ tend to work in opposition. This results in the following scenario when $B$ is changed. When $\mu$ lies in the region of localized states below a LL band, $\rho_{21}$ is very small because very few electrons have sufficient energy to be excited into extended states where they contribute to the drag [23]. As $B$ is increased so that $\mu$ moves into the LL band, the density of extended states increases, while the interlayer interaction is strong due to weak screening, resulting in a sharp rise in $\rho_{21}$. However, as the $B$ field is further increased so that $\mu$ moves closer towards the center of the LL and the DOS further increases, the screening becomes so effective that it more than compensates for the increase in DOS, leading to a reduction in $\rho_{21}$. This competition of DOS and screening produces the unique shape of $\rho_{21}(B)$.

We also find interesting behavior in $\rho_{21}$ when $B$ is kept constant and the temperature $T$ is changed. We concentrate on the $T$ dependence for $\mu$ in the middle of a

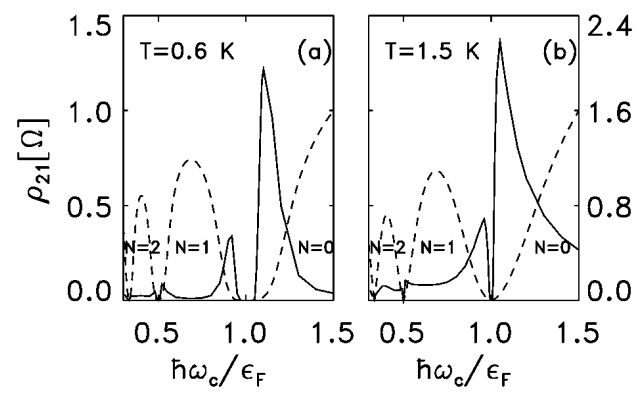

FIG. 3. Transresistivity $\rho_{21}$ (solid lines) and the thermally averaged DOS $g=\partial n / \partial \mu$ (dashed, in arbitrary units) for (a) $T=0.6 \mathrm{~K}$ and (b) $T=1.5 \mathrm{~K}$ as a function of magnetic field in GaAs for density $n=1.5 \times 10^{11} \mathrm{~cm}^{-2}\left(T_{F}=\right.$ $\left.E_{F} / k_{B} \approx 60 \mathrm{~K}\right)$, well separation $d=350 \AA$, zero well widths, and $\hbar \tau^{-1}=0.01 E_{F} . \quad N$ is the LL index, and $\hbar \omega_{c}=\epsilon_{F}$ corresponds to $B=3.1 \mathrm{~T}$. While the $g(B)$ peaks in the middle of the Landau level, the interlayer coupling is weakest there (due to large screening), pushing the peaks in $\rho_{21}$ towards the edges of the Landau level bands.
LL band. If the DOS were constant and the interaction were $\omega$ independent, the scaled transresistivity $\rho_{21} / T^{2}$ would be $T$ independent [7]. Figure 4, however, shows that $\rho_{21} / T^{2}$ has two prominent features: (1) a $\log (T)$ behavior for $T \ll \tau^{-1}=0.01 E_{F}$ (see inset), and (2) an enhancement due to dynamic screening effects.

The logarithmic behavior of $\rho_{21} / T^{2}$, which is a direct consequence of the diffusive nature of the system [8], can be seen at experimentally feasible temperatures $(T \sim$ $0.6 \mathrm{~K}$, see inset of Fig. 4) at high $B$ fields, in sharp contrast to the $B=0$ case, where it manifests itself only at unattainably low temperatures (for high-mobility samples Ref. [8] finds $T \sim 10^{-100} \mathrm{~K}$ ). This difference can be traced to the different length scales over which diffusive behavior occurs. At $B=0$, the minimum length scale for diffusive behavior to be seen is the elastic mean free path $l_{\mathrm{el}}=v_{F} \tau$, whereas at high $B$ fields this length scale is $\ell_{B}$, since diffusion occurs by hopping from adjacent orbitals which are $\ell_{B}$ apart. This implies that the diffusive form of the polarizability $\chi_{\mathrm{diff}}(q, \omega) \sim$ $-D q^{2} /\left(D q^{2}-i \omega\right)$ is valid for $q \lesssim l_{\mathrm{el}}^{-1}$ for $B=0$ and $q \lesssim \ell_{B}^{-1}$ for large $B$. Since $l_{\mathrm{el}} \gg \ell_{B}$ at large $B$, it is much easier to pick up the diffusive behavior at high magnetic fields. The second feature noted is a dynamic screening induced enhancement in $\rho_{21} / T^{2}$, reminiscent of an effect occurring at $B=0$ [12]. As the temperature is raised, inelastic interlayer momentum transfer processes become increasingly important. These processes are screened dynamically, and as this is less effective than static screening, the effective interlayer interaction is increased leading to an enhanced $\rho_{21} / T^{2}$.

Summarizing, we have presented a microscopic calculation of transresistivity for Coulomb coupled quantum wells in strong magnetic fields. Both magnetic field and temperature dependence of the transresistivity are clearly distinct from normal longitudinal magnetoresistivity; the

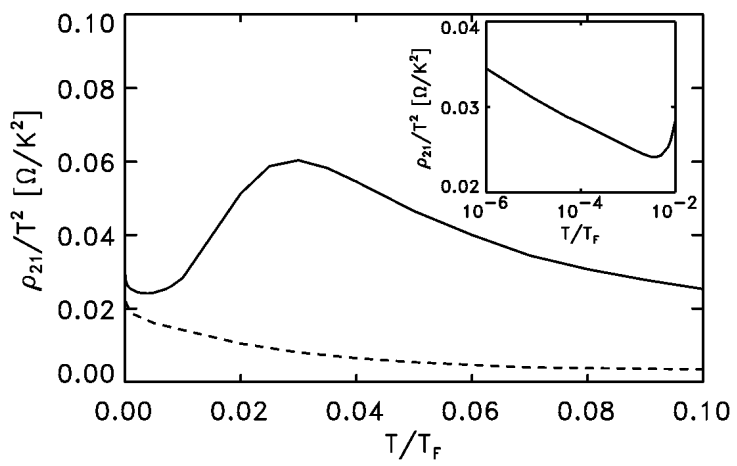

FIG. 4. Transresistivity as a function of temperature, for $B=$ $2.1 \mathrm{~T}$ (equivalent to a half filled $N=1 \mathrm{LL}$ ), using dynamical screening (solid line) and, for comparison, static screening [i.e., using $W_{12}(q, \omega=0)$, dashed line]. Other parameters are as in Fig. 3. The upturn and maximum in the solid curve is caused by the decrease in the screening efficiency of the electron gas at finite frequencies. Inset: Closeup of dynamical screening curve for $10^{-6}<T / T_{F}<10^{-2}$, showing $\rho_{21} / T^{2} \sim-\log (T)$. 
differences arise from an intricate interplay between Landau quantization, interparticle interaction, and diffusion effects.

We thank A. H. MacDonald and A. Wacker for useful comments, and Nicholas Hill for sharing his unpublished experimental results with us. K.F. was partially supported by the Carlsberg Foundation.

[1] See, e.g., The Quantum Hall Effect, edited by R. E. Prange and S.M. Girvin (Springer-Verlag, New York, 1990), 2nd ed.

[2] D. B. Chklovskii, B. I. Shklovskii, and L. I. Glazman, Phys. Rev. B 46, 4026 (1992).

[3] W. Kohn, Phys. Rev. 123, 1242 (1961); L. Brey, N. F. Johnson, and B.I. Halperin, Phys. Rev. B 40, 10647 (1989).

[4] J. P. Eisenstein, L. N. Pfeiffer, and K. W. West, Phys. Rev. Lett. 69, 3804 (1992); S. Q. Murphy, J. P. Eisenstein, and K. W. West, Phys. Rev. B 52, 14825 (1995).

[5] P. M. Solomon, P. J. Price, D. J. Frank, and D. C. La Tulipe, Phys. Rev. Lett. 63, 2508 (1989); T. J. Gramila, J.P. Eisenstein, A.H. MacDonald, L. N. Pfeiffer, and K. W. West, Phys. Rev. Lett. 66, 1216 (1991); Phys. Rev. B 47, 12957 (1993); Physica (Amsterdam) 197B, 442 (1994); U. Sivan, P. M. Solomon, and H. Shtrikman, Phys. Rev. Lett. 68, 1196 (1992).

[6] B. Laikhtman and P.M. Solomon, Phys. Rev. B 41, 9921 (1990); I. I. Boiko and Yu. M. Sirenko, Phys. Status Solidi 159, 805 (1990); P. M. Solomon and B. Laikhtman, Superlattices Microstruct. 10, 89 (1991); A. G. Rojo and G. D. Mahan, Phys. Rev. Lett. 68, 2074 (1992); H.C. Tso, P. Vasilopoulos, and F. M. Peeters, Phys. Rev. Lett. 68, 2516 (1992); 70, 2146 (1993); H. C. Tso and P. Vasilopoulos, Phys. Rev. B 45, 1333 (1992); D. I. Maslov, Phys. Rev. B 45, 1911 (1992); J.-M. Duan and S. Yip, Phys. Rev. Lett. 70, 3647 (1993); H. L. Cui, X. L. Lei, and N. J. M. Horing, Superlattices Microstruct. 13, 221 (1993); K. Flensberg and B. Y.-K. Hu, Phys. Rev. Lett. 73, 3572 (1994); L. Świekowski, J. Szymański, and Z.W. Gortel, Phys. Rev. Lett. 74, 3245 (1995); Ji-Min Duan, Europhys. Lett. 29, 489 (1995).

[7] A.-P. Jauho and H. Smith, Phys. Rev. B 47, 4420 (1993).

[8] L. Zheng and A.H. MacDonald, Phys. Rev. B 48, 8203 (1993).

[9] E. Shimshoni and S. L. Sondhi, Phys. Rev. B 49, 11484 (1994).

[10] A. Kamenev and Y. Oreg, Phys. Rev. B 52, 7516 (1995).

[11] K. Flensberg, B. Y.-K. Hu, A.-P. Jauho, and J. Kinaret, Phys. Rev. B 52, 14761 (1995).

[12] K. Flensberg and B. Y.-K. Hu, Phys. Rev. B 52, 14796 (1995).
[13] The transresistivity is related to the drag rate $\tau_{D}^{-1}$, which is defined and used in several papers in Refs. [5] and [6], by $\rho_{21}^{\alpha \alpha}=m_{1} / n_{1} e^{2} \tau_{D}$.

[14] This form is for a uniform system, and ignores edge effects. We expect this form to be valid in this paper since the significant results we present are for the interplateau regions where the eigenstates are extended and bulklike.

[15] T. Ando, A. B. Fowler, and F. Stern, Rev. Mod. Phys. 54, 437 (1982), and references therein.

[16] M. M. Fogler and B. I. Shklovskii, Phys. Rev. B 52, 17366 (1995).

[17] The 3 vertex corrections and $3 G$ 's in $\boldsymbol{\Delta}$ are reduced to 1 vertex and $2 G$ 's in $\chi$ as follows. (i) Vertices: When $\omega_{c} \tau \gg 1$, vertex corrections are significant only when the incoming LL indices are equal. The $\gamma$ can be neglected because the LL indices of the incoming $G$ 's differ by 1 , and of the remaining $\Gamma$ 's, only one can have equal incoming indices. (ii) Green functions: $G(N, \omega) G(N-1, \omega) \approx[G(N, \omega)-$ $G(N-1, \omega)] / \omega_{c}+O\left(\left(\omega_{c} \tau\right)^{-1}\right)$, reducing $3 G$ 's to 2. This creates "mismatches" between the LL indices of the bare vertex functions $f_{M, N}(\mathbf{q}) \equiv \mid\left\langle m, k_{y}\right| \times$ $\exp (-i \mathbf{q} \cdot \mathbf{x})\left|n, k_{y}+q_{y}\right\rangle \mid$ and the $G$ 's [i.e., it produces $G(M) G(N) f_{M, N \pm 1}$; the matches are restored and a factor of $\mathbf{q}$ created with the identity $\sqrt{N+1} f_{N+1, M}(\mathbf{q})-\sqrt{M} f_{N, M-1}(\mathbf{q}) \equiv-\ell_{B}\left(i q_{x}+\right.$ $\left.q_{y}\right) 2^{-1 / 2} f_{N, M}(\mathbf{q})-1$, where $\ell_{B}=(\hbar / e B)^{1 / 2}$ is the magnetic length.

[18] This can be understood physically by considering the semiclassical electron distribution function. The relationship $\Delta \propto \operatorname{Im}[\chi]$ results when the distribution function in the linear current response regime is a drifted Fermi-Dirac, which occurs both for constant $U$ at $B=0$ and for arbitrary $U(q)$ at large $B$ fields.

[19] M. Bønsager, K. Flensberg, B. Y.-K. Hu, and A.-P. Jauho (unpublished).

[20] See, e.g., D. Pines and P. Nozières, The Theory of Quantum Liquids (Benjamin, New York, 1966), Vol. 1, Eqs. (4.26) and (4.60).

[21] This is in qualitative agreement with recent unpublished experiments [N. Hill (private communication)].

[22] We assume that all the electrons in the SCBA LL bands are extended and insert localized states (which do not contribute to transport properties such as $\rho_{21}$ ) in between the LL bands "by hand" to mimic experimental conditions. Note that our theory breaks down below $200 \mathrm{mK}$, when localization effects begin to play an important role throughout the LL band; see H.P. Wei et al., Phys. Rev. B 45, 3926 (1992).

[23] While we cannot say anything quantitative about the quantum Hall plateau regime because our model excludes some of the pertinent physics here, we expect $\rho_{21}$ to be small because there is an energy gap to excitations. 